\title{
Comunicação
}

[Communication]

\section{Efeitos de Beauveria bassiana (Bals) Vuill e Metarhizium anisopliae (Metsc) Sorok sobre fêmeas ingurgitadas de Amblyomma cajennense (Fabricius, 1787) em condições de laboratório}

[Effects of Beauveria bassiana (Bals) Vuill and Metarhizium anisopliae (Metsc) Sorok on engorged females of Amblyomma cajennense (Fabricius, 1787) in laboratory conditions]

\author{
R.C.S. Reis ${ }^{1}$, D. R. Melo ${ }^{1}$, V.R.E.P. Bittencourt ${ }^{2,3 *}$ \\ ${ }^{1}$ Curso de Pós-graduação em Ciências Veterinárias - UFRRJ - Bolsista da CAPES \\ ${ }^{2}$ Departamento de Parasitologia Animal - Instituto de Veterinária da UFRRJ \\ $\mathrm{Br} 465, \mathrm{~km} 7$ \\ 23890-000 - Seropédica, RJ \\ ${ }^{3}$ Bolsista do $\mathrm{CNPq}$
}

\begin{abstract}
Amblyomma cajennense (Fabricius, 1787), conhecido popularmente como carrapato estrela, é um carrapato heteroxeno, pertencente à família Ixodidae e subfamília Amblyomminae. É encontrado principalmente em eqüídeos, mas pode também se alimentar em bovinos e cães, ou no próprio homem (Flechman, 1985). A espécie, de ampla distribuição geográfica no continente americano, ocorre desde o Texas, nos Estados Unidos, até a América do Sul, concentrando-se ao longo da costa do Oceano Atlântico (Robinson, 1926). Segundo Serra Freire (1982), o Brasil, em decorrência de suas variáveis climáticas, é uma região favorável ao desenvolvimento de ixodídeos em fase de vida livre durante todo o ano.
\end{abstract}

As infestações por carrapatos em animais domésticos são controladas principalmente por acaricidas químicos. Quando utilizados de forma indiscriminada podem causar impacto ambiental e desenvolvimento de resistência (Bittencourt et al., 1997). Segundo Barros e Evans (1989), o uso exclusivo de carrapaticidas é pouco viável em termos práticos e econômicos, tornando-se necessário o emprego de métodos alternativos em sistemas de controle integrado.
Pesquisas têm sido realizadas com o objetivo de usar patógenos como controladores biológicos, visando estabelecer melhores estratégias de combate ao parasito. Fungos entomopatogênicos são importantes inimigos naturais de artrópodes e podem ser utilizados no seu controle biológico (Chandler et al., 2000). Aproximadamente 750 espécies e 56 gêneros são conhecidos por serem patógenos ou parasitos de artrópodes (Hawksworth, 1977). A patogenicidade do fungo Metarhizium anisopliae foi verificada em fêmeas ingurgitadas de Boophilus microplus (Bittencourt, 1992), nas quais se constatou elevada mortalidade e controle de $96,6 \%$ do isolado 959. A ação in vitro do fungo Beauveria bassiana para ovos da mesma espécie de carrapato também foi avaliada por Bittencourt et al. (1996). Os autores observaram que o percentual de eclosão verificado nos grupos tratados foi menor que o observado nos grupos controle.

O objetivo do presente trabalho foi avaliar as alterações ocorridas no ciclo biológico de fêmeas ingurgitadas de $A$. cajennense quando submetidas a tratamentos com os fungos $M$. anisopliae e $B$. bassiana mantidos sob condições de laboratório.

Recebido para publicação em 12 de dezembro de 2003

Recebido para publicação, após modificações, em 12 de maio de 2004

*Autor para correspondência

E-mail: vaniabit@ufrrj.br 
Os isolados de $M$. anisopliae e $B$. bassiana foram cedidos pelo Departamento de Entomologia da Escola Superior de Agricultura "Luiz de Queiroz", da Universidade de São Paulo. Os isolados foram de $M$. anisopliae, denominados Ma 319, Ma 959 e Ma E9, e de B. bassiana, Bb 747 e $\mathrm{Bb}$ 986. Mantidos e reproduzidos no laboratório de controle microbiano de artrópodes de importância veterinária da Universidade Federal do Rio de Janeiro, os fungos foram produzidos em meio de arroz, em sacos de polipropileno para a produção das suspensões. Uma porção de arroz contendo o fungo foi homogeneizada em becker graduado de $100 \mathrm{ml}$ contendo água destilada com $0,1 \%$ de espalhante adesivo Tween $80^{\circledR}$. Após homogeneização, uma amostra da suspensão foi colocada em câmara de Neubauer e quantificada ao microscópio para avaliação do número de conídios. As suspensões foram preparadas em quatro concentrações crescentes de $10^{5}$ a $10^{8}$ conídios por mililitro de suspensão, além do grupo-controle, composto de $100 \mathrm{ml}$ de água destilada e $0,1 \%$ de espalhante adesivo.

As fêmeas ingurgitadas utilizadas foram obtidas a partir de infestações experimentais em áreas delimitadas em eqüinos, segundo a técnica de Prata et al. (1995). Cada animal foi infestado com 160 casais adultos de $A$. cajennense, com aproximadamente 20 dias em jejum, para a obtenção das amostras. As fêmeas ingurgitadas de $A$. cajennense, coletadas 10 a 15 dias após a infestação artificial, foram levadas ao laboratório, lavadas em solução de hipoclorito de sódio a $1 \%$, enxaguadas em água estéril por um minuto, secas em papel filtro para a retirada de excesso de água, identificadas, pesadas e separadas em tubos de ensaios individualizados estéreis para a infecção com os isolados. As suspensões foram utilizadas para a imersão das fêmeas ingurgitadas de $A$. cajennense, baseada nas técnicas de avaliação de carrapaticidas descritas por Torrado e Gutierrez (1969) e Stendel (1980). Após a imersão durante cinco minutos, foram acondicionadas em placas de Petri, em alíquotas de 10 indivíduos por placa. As do grupo-controle foram banhadas por cinco minutos em água destilada acrescido de espalhante adesivo Tween $80^{\circledR}$ a $1 \%$.

Foram realizadas 10 repetições por tratamento, resultando em 500 fêmeas ingurgitadas. Elas foram fixadas em decúbito dorsal na placa de
Petri, com o auxílio de uma fita adesiva dupla face, para manter individualizada a coleta diária das massas de ovos e facilitar a identificação de cada fêmea.

As características avaliadas foram: peso e período de postura, índice de eficiência reprodutiva (IER), índice de eficiência nutricional (IEN) e percentual de controle, calculados segundo Bennett (1974). Foram realizadas análises de próbites para calcular as concentrações letais, CL 50 e CL 90, segundo Litchfield e Wilcoxon (1949). Para verificar se houve variações dentro de um mesmo tratamento e entre tratamentos com os diferentes isolados e concentrações, foram realizadas análises de variância seguidas de aplicação do teste Tukey, para comparação de médias.

Os resultados dos tratamentos com os fungos são apresentados na Tab. 1. Nas concentrações mais elevadas foram observadas diferenças em relação ao grupo-controle para todas as características avaliadas. O isolado Ma 319 apresentou o menor peso da massa de ovos $(79,1 \pm 33,6 \mathrm{mg})$ em relação ao grupo-controle $(323,8 \pm 75,6 \mathrm{mg})$, isto é, este isolado foi mais patogênico nas condições avaliadas. Os isolados MA 959 e Ma E9 apresentaram períodos de postura mais curtos que os demais isolados.

Para o IEN e o IER foram observadas diminuições significativas nos grupos tratados com todos os isolados. Esses índices estimam a capacidade do carrapato de transformar nutrientes em ovos; quando seu valor é baixo indica que a fêmea realizou postura menor do que é capaz. Neste trabalho, os índices diminuíram à medida que se aumentou a concentração de conídios/ml de suspensão. Ocorreram diferenças significativas entre os isolados utilizados na maioria dos bioensaios quando comparados com o grupo-controle, constatando que a infecção com as diversas concentrações foi capaz de alterar o ciclo reprodutivo. Bittencourt (1992), ao avaliar o efeito do fungo $M$. anisopliae no carrapato $B$. microplus, observou diminuição do período de postura de fêmeas ingurgitadas tratadas. Essa diminuição também foi constatada por Monteiro (2000), ao estudar fêmeas ingurgitadas de Anocentor nitens expostas ao isolado 986 do fungo $B$. bassiana. Os dados apresentados neste trabalho assemelham-se aos dos autores citados. 
Tabela 1. Período de postura, peso de postura, índice de eficiência nutricional (IEN), índice de eficiência reprodutiva (IER) e percentual de controle (\%) de fêmeas de Amblyomma cajennense em diversas concentrações dos isolados de Metarhizium anisopliae (Ma) e Beauveria bassiana (Bb)

\begin{tabular}{lcccccc}
\hline Característica & Con/ml* & Ma 959 & Ma 319 & Ma E9 & Bb 747 & Bb 986 \\
\hline & $\mathrm{C}$ & $23,4 \pm 3,5 \mathrm{a}$ & $23,4 \pm 3,5 \mathrm{a}$ & $23,4 \pm 3,5 \mathrm{a}$ & $23,4 \pm 3,5 \mathrm{a}$ & $23,4 \pm 3,5 \mathrm{a}$ \\
Período de postura & $10^{5}$ & $18,4 \pm 8,0 \mathrm{a}$ & $18,2 \pm 8,4 \mathrm{ab}$ & $19,9 \pm 3,0 \mathrm{ab}$ & $16,5 \pm 4,4 \mathrm{~b}$ & $13,8 \pm 5,7 \mathrm{~b}$ \\
& $10^{6}$ & $13,6 \pm 2,2 \mathrm{a}$ & $17,6 \pm 6,9 \mathrm{bc}$ & $18,2 \pm 6,6 \mathrm{ab}$ & $14,3 \pm 2,5 \mathrm{bc}$ & $12,8 \pm 5,9 \mathrm{~b}$ \\
& $10^{7}$ & $9,1 \pm 7,1 \mathrm{a}$ & $14,6 \pm 9,8 \mathrm{bc}$ & $6,7 \pm 2,6 \mathrm{c}$ & $13,3 \pm 3,0 \mathrm{bc}$ & $9,3 \pm 1,6 \mathrm{~b}$ \\
& $10^{8}$ & $5,3 \pm 2,7 \mathrm{~b}$ & $8,4 \pm 6,5 \mathrm{c}$ & $4,2 \pm 1,5 \mathrm{bc}$ & $11,7 \pm 4,0 \mathrm{c}$ & $11,6 \pm 8,2 \mathrm{~b}$ \\
Peso de postura & $\mathrm{C}$ & $323,8 \pm 75,6 \mathrm{a}$ & $323,8 \pm 75,6 \mathrm{a}$ & $323,8 \pm 75,6 \mathrm{a}$ & $323,8 \pm 75,6 \mathrm{a}$ & $323,8 \pm 75,6 \mathrm{a}$ \\
& $10^{5}$ & $291,8 \pm 68,5 \mathrm{a}$ & $283,7 \pm 89,4 \mathrm{a}$ & $264,2 \pm 60,0 \mathrm{ab}$ & $285,4 \pm 63,5 \mathrm{a}$ & $137,9 \pm 76,3 \mathrm{~b}$ \\
& $10^{6}$ & $286,1 \pm 59,3 \mathrm{a}$ & $115,5 \pm 82,5 \mathrm{~b}$ & $219,0 \pm 71,6 \mathrm{~b}$ & $258,9 \pm 50,8 \mathrm{~b}$ & $128,4 \pm 64,6 \mathrm{~b}$ \\
& $10^{7}$ & $21,6 \pm 91,5 \mathrm{a}$ & $114,0 \pm 54,6 \mathrm{~b}$ & $177,2 \pm 84,3 \mathrm{c}$ & $168,0 \pm 57,6 \mathrm{~b}$ & $129,0 \pm 75,6 \mathrm{~b}$ \\
IEN & $10^{8}$ & $160,4 \pm 67,3 \mathrm{~b}$ & $79,1 \pm 33,6 \mathrm{c}$ & $153,1 \pm 80,9 \mathrm{c}$ & $163,9 \pm 49,0 \mathrm{~b}$ & $113,2 \pm 48,3 \mathrm{~b}$ \\
& $\mathrm{C}$ & $68,05 \pm 27,1 \mathrm{a}$ & $68,05 \pm 27,1 \mathrm{a}$ & $68,05 \pm 27,1 \mathrm{a}$ & $68,05 \pm 27,1 \mathrm{a}$ & $68,05 \pm 27,1 \mathrm{a}$ \\
& $10^{5}$ & $50,77 \pm 18,3 \mathrm{a}$ & $59,35 \pm 6,4 \mathrm{a}$ & $66,61 \pm 12,7 \mathrm{a}$ & $49,54 \pm 21,9 \mathrm{a}$ & $57,71 \pm 15,8 \mathrm{a}$ \\
& $10^{6}$ & $54,56 \pm 16,1 \mathrm{a}$ & $44,24 \pm 29,5 \mathrm{~b}$ & $66,73 \pm 16,4 \mathrm{a}$ & $39,36 \pm 17,0 \mathrm{~b}$ & $44,28 \pm 19,1 \mathrm{a}$ \\
IER & $10^{7}$ & $49,71 \pm 20,3 \mathrm{~b}$ & $42,57 \pm 26,6 \mathrm{~b}$ & $31,64 \pm 10,81 \mathrm{c}$ & $34,18 \pm 11,9 \mathrm{~b}$ & $38,96 \pm 12,7 \mathrm{~b}$ \\
& $10^{8}$ & $20,60 \pm 12,6 \mathrm{c}$ & $18,12 \pm 13,7 \mathrm{c}$ & $28,00 \pm 13,9 \mathrm{c}$ & $23,80 \pm 14,1 \mathrm{c}$ & $26,80 \pm 14,1 \mathrm{~b}$ \\
& $\mathrm{C}$ & $52,83 \pm 17,2 \mathrm{a}$ & $52,83 \pm 17,2 \mathrm{a}$ & $52,83 \pm 17,2 \mathrm{a}$ & $52,83 \pm 17,2 \mathrm{a}$ & $52,83 \pm 17,2 \mathrm{a}$ \\
& $10^{5}$ & $51,92 \pm 11,3 \mathrm{ab}$ & $41,93 \pm 12,4 \mathrm{a}$ & $45,56 \pm 19,2 \mathrm{ab}$ & $45,53 \pm 22,2 \mathrm{a}$ & $28,55 \pm 12,0 \mathrm{~b}$ \\
& $10^{6}$ & $42,01 \pm 12,3 \mathrm{~b}$ & $27,47 \pm 17,4 \mathrm{~b}$ & $40,17 \pm 15,6 \mathrm{ab}$ & $44,16 \pm 17,3 \mathrm{a}$ & $25,14 \pm 15,0 \mathrm{~b}$ \\
& $10^{7}$ & $35,37 \pm 15,8 \mathrm{~b}$ & $24,25 \pm 10,1 \mathrm{bc}$ & $35,37 \pm 15,3 \mathrm{~b}$ & $38,24 \pm 19,7 \mathrm{~b}$ & $19,03 \pm 11,3 \mathrm{c}$ \\
& $10^{8}$ & $18,68 \pm 7,2 \mathrm{c}$ & $13,22 \pm 7,2 \mathrm{c}$ & $8,68 \pm 1,8 \mathrm{c}$ & $20,76 \pm 11,4 \mathrm{c}$ & $15,83 \pm 10,2 \mathrm{c}$
\end{tabular}

Médias seguidas de letras distintas na coluna diferem entre si pelo teste Tukey $(\mathrm{P}<0,05)$

*Concentração de conídios/ml.

O percentual de controle evidenciou números acima de $90 \%$ para os três isolados de $M$. anisopliae, o que não foi observado para os isolados de B. bassiana. Segundo Bittencourt et al. (1997), os isolados 986 e 747 do fungo $B$. bassiana apresentaram controle de $B$. microplus de $59,9 \%$ a $88,3 \%$ e $63,7 \%$ a $83,4 \%$, respectivamente. Segundo Bittencourt (1992), o controle de B. microplus ao isolado Ma 959 na concentração $10^{8}$ conídios $/ \mathrm{ml}$ foi de $96,6 \%$. Os resultados do presente trabalho assemelham-se aos desses autores, isto é, ocorreu aumento do índice à medida que se aumentou a concentração de conídios $/ \mathrm{ml}$. A concentração $10^{8}$ conídios $/ \mathrm{ml}$ do isolado Ma 959 apresentou o maior índice de controle, 97,8\%. Estes resultados reforçam a hipótese de que esses patógenos são capazes de promover efeitos deletérios à biologia do $A$. cajennense em ensaios in vitro, e têm grande importância no seu controle, pela possibilidade de redução da taxa de crescimento do carrapato no campo.

Palavras-chave: carrapato, Amblyomma cajennense, controle biológico, fungo

\section{ABSTRACT}

The in vitro susceptibility of Amblyomma cajennense engorged females to some isolated of the fungus Beauveria bassiana and Metarhizium anisopliae was verified and lethal concentrations ( $L C 50$ and LC 90) were calculated. The females were dived in conidia suspensions for five minutes, and kept in climatically controlled chambers BOD under $27^{\circ} \mathrm{C}$ and $80 \%$ relative humidity. Each bioassay had four 
treatments in concentrations of $10^{5}, 10^{6}, 10^{7}$ e10 conidia/ml. A control group was also used. The following characteristics were evaluated: weight and period of oviposition, indexes of reproductive and nutritional efficiency and percentage of microbiological control. A dose dependent negative effect was observed in ticks treated with the suspension. All isolates tested cause a negative effect on in vitro tests of engorged females of A. cajennense, suggesting its potential for microbiological control of tick's species.

Keywords: tick, Amblyomma cajennense, biological control, fungus

\section{REFERÊNCIAS BIBLIOGRÁFICAS}

BARROS, T.A.M.; EVANS, D.E. Ação de gramíneas forrageiras em larvas infestantes do carrapato dos bovinos, Boophilus microplus. Pesq. Vet. Bras., v.9, p.17-21, 1989.

BENNETT, G.F. Oviposition of Boophiluis microplus (Canestrini) (Acarida: Ixodidae). I. Influence of tick size on egg production. Acarologia, v.16, p.52-61, 1974.

BITTENCOURT, V.R.E.P. Ação do fungo Metarhizium anisopliae (Metchnikoff, 1879) Sorokin, 1883, sobre o carrapato Boophilus microplus (Canestrini, 1887). 1992. 105f. Tese (Doutorado) - Universidade Federal Rural do Rio de Janeiro, Seropédica, RJ.

BITTENCOURT, V.R.E.P.; SOUZA, E.J.; PERALVA, S.L.F.S. et al. Avaliação dos efeitos do contato de Beauveria bassiana (Bals.) Vuill. com ovos e larvas de Boophilus microplus (Canestrini, 1887) (Acari: Ixodidae). Rev. Bras. Parasitol. Vet., v.5, p.81-84, 1996.

BITTENCOURT, V.R.E.P.; SOUZA, E.J.; PERALVA, S.L.F.S. et al. Avaliação da eficácia in vitro do fungo entomopatogênico Beauveria bassiana (Bals.) Vuill. em fêmeas ingurgitadas de Boophilus microplus (Canestrini, 1887) (Acari:Ixodidae). Rev. Bras. Parasitol. Vet., v.6, p.49-52, 1997.

CHANDLER, D.; DAVIDSON, G.; PELL, J.K. et al. Fungal biocontrol of acari. Biocontrol Sch. Technol., v.10 p.357-384, 2000.

FLECHTMANN, C.H.W. Ácaros de importância médico-veterinária. 3.ed. São Paulo: Editora Nobel, 1985. 192p.
HAWKSWORTH, D.L. Mycologist's handbook. 2.ed. Kew Surrey, England: CAB, 1977. 231p.

LITCHFIELD, J.T.; WILCOXON, F. Simple method of fitting dose effect curve. J. Pharm. Exp. Ther., v.95, p.99-113, 1949.

MONTEIRO, S.G. Ação do isolado 986 do fungo Beauveria bassiana (Balsamo) Vuillemin, 1912, sobre o carrapato Anocentor nitens (Neumann) Schulze, 1937. 2000. 75f. Tese (Doutorado) - Universidade Federal Rural do Rio de Janeiro, Seropédica, RJ.

PRATA, M.C.A.; MORAIS, M.C.; ALONSO, L.S. et al. Determinação de alguns parâmetros biológicos de Amblyomma cajennense (Fabricius, 1787) (Acari: Ixodidae) em infestações artificiais de eqüinos. Rev. Bras. Parasit. Vet., v.4, supl., p.1-49, 1995.

ROBINSON, L.E. The genus Amblyomma. Cambridge University, 1926. 301p.

SERRA FREIRE, N.M. Epidemiologia de Amblyomma cajennense: ocorrência estacional e comportamento dos estádios não parasitários em pastagens do Estado do Rio de Janeiro. Arq. Univ. Fed. Rural Rio de Janeiro, v.5, p.187-193, 1982.

STENDEL, W. The relevance of different test methods for the evaluation of tick controlling substances. J. South Afr. Vet. Assoc., v.51, p.147$152,1980$.

TORRADO, J.M.G.; GUTHIERREZ, R.O. Metodo para medir la actividad de los acaricidas sobre larvas de garrapata. Evolucion de sensibilidad. Rev. Inst. Agr. Patol., v.6, p.135158,1969 